\title{
Electronic and excitonic properties of self-assembled semiconductor quantum rings
}

\author{
V. M. Fomin ${ }^{a, b, c, d *}$, V. N. Gladilin ${ }^{b, d, e}$, J. T. Devreese ${ }^{b, c}$, \\ J. H. Blokland ${ }^{f}$, P. C. M. Christianen $f$, J. C. Maan ${ }^{f}$, \\ A. G. Taboada ${ }^{g}$, D. Granados $^{g}$, J. M. García ${ }^{g}$, \\ N. A. J. M. Kleemans ${ }^{c}$, H. C. M. van Genuchten ${ }^{c}$, M. Bozkurt ${ }^{c}$ and P. M. $\operatorname{Koenraad}^{c}$ \\ ${ }^{a}$ Present address: Theoretische Physik and Center for Nanointegration Duisburg-Essen, \\ Universität Duisburg-Essen, Lotharstr. 1, D-47058 Duisburg, Germany; \\ ${ }^{b}$ Theoretische Fysica van de Vaste Stoffen, Universiteit Antwerpen, \\ Groenenborgerlaan 171, B-2020 Antwerpen, Belgium; \\ ${ }^{c}$ Photonics and Semiconductor Nanophysics, COBRA, TU Eindhoven, \\ Den Dolech 2, Postbus 513, NL-5600 MB Eindhoven, The Netherlands; \\ ${ }^{d}$ Also at: Physics of Multilayer Structures and Molecular Magnetism, \\ State University of Moldova, str. A. Mateevici 60, MD-2009 Chişinău, Moldova; \\ ${ }^{e}$ Present address: Institute for Nanoscale Physics and Chemistry, \\ Katholieke Universiteit Leuven, Celestijnenlaan 200D, B-3001 Leuven, Belgium; \\ ${ }_{\text {HFML, IMM, Radboud University Nijmegen, }}$ \\ Toernooiveld 7, NL-6525 ED Nijmegen, The Netherlands; \\ ${ }^{g}$ CNM-CSIC, Instituto de Microelectrónica de Madrid, \\ C/Isaac Newton 8, Tres Cantos, E-28760 Madrid, Spain
}

\begin{abstract}
Theoretical analysis of the electron energy spectrum and the magnetization in a strained $\operatorname{In}_{x} \mathrm{Ga}_{1-x} \mathrm{As} / \mathrm{GaAs}$ selfassembled quantum ring (SAQR) is performed using realistic parameters, determined from the cross-sectional scanning-tunneling microscopy characterization. The Aharonov-Bohm oscillations in the persistent current have been observed in low temperature magnetization measurements on these SAQRs. The effect of the Coulomb interaction on the energy spectra of SAQRs is studied for rings with two electrons and with an exciton. Our analysis of the photoluminescence spectrum in magnetic fields up to $30 \mathrm{~T}$ shows that the excitonic properties strongly depend on the anisotropic shape, size, composition and strain of the SAQRs and is in a good agreement with the experimental data.
\end{abstract}

Keywords: Self-assembled semiconductor quantum rings, magnetization, photoluminescence, Aharonov-Bohm effect, persistent currents

\section{INTRODUCTION}

Electrons confined to a small ring manifest their quantum nature by an oscillatory behaviour of their energy levels as a function of an applied magnetic field. This effect originates from the periodic dependence of the phase of the electron wave function on the magnetic flux through the ring, the Aharonov-Bohm effect. ${ }^{1}$ Theoretical analysis of the electron energy spectrum and the magnetization of an electron in a strained $\operatorname{In}_{x} \mathrm{Ga}_{1-x} \mathrm{As} / \mathrm{GaAs}$ self-assembled quantum ring (SAQR) is performed with realistic parameters, determined from the cross-sectional scanning-tunneling microscopy characterization of that nanostructure. The SAQRs have an asymmetric indiumrich crater-like shape with a depression rather than an opening at the center. Although the real SAQR shape differs strongly from an idealized circular-symmetric open ring structure, the Aharonov-Bohm oscillations of the

*Correspondence should be addressed to V. M. Fomin.

E-mail: vladimir.fomin@uni-due.de, Telephone: +49 203379 4744, Fax: +49 2033794732.

Nanotechnology IV, edited by Achim Wixforth, Axel Lorke, Friedrich C. Simmel, Proc. of SPIE Vol. 7364,

$736402 \cdot$ C 2009 SPIE · CCC code: 0277-786X/09/\$18 · doi: 10.1117/12.822607

Proc. of SPIE Vol. 7364 736402-1 
magnetization survive. ${ }^{2}$ The Aharonov-Bohm oscillations in the persistent current have been observed in low temperature magnetization measurements on $\operatorname{In}_{x} \mathrm{Ga}_{1-x} \mathrm{As} / \mathrm{GaAs}$ SAQRs. ${ }^{3}$ The effect of the Coulomb interaction on the energy spectra of SAQRs is studied for rings with two electrons and with an exciton. With increasing magnetic field, the lowest spin-singlet and spin-triplet states in a two-electron SAQR sequentially replace each other as the ground state. ${ }^{4}$

Formation of ring-shaped excitons for an antidot in a 2D electron gas due to the skipping states of electrons and holes in a strong perpendicular magnetic field was theoretically investigated in Ref. ${ }^{6}$ The ground state energy of such an exciton was shown to be logarithmically larger as compared to that for a $2 \mathrm{D}$ exciton in the bulk. The possibility of tunneling of the electron to the hole (or vice versa) around the antidot shifts the energy levels from those for the bulk 2D excitons and leads to the periodic dependence of the binding energy on the magnetic flux with the period $\Phi_{0}$. Subsequently, there have been several theoretical works on possible mechanisms leading to the Aharonov-Bohm oscillations in the optical properties of neutral excitons in quantum rings ${ }^{7-11}$ and quantum dots. ${ }^{12}$ One mechanism is due to the fact that in a ring-like nanostructure electrons and holes have different trajectories, what leads to the occurrence of a nonzero electric dipole moment (or, in other words, "polarization") of an exciton. Another mechanism is due to the strong spatial separation of the electron and the hole in type-II quantum dots, which leads to an enhanced polarization of excitons.

The optical absorption spectra observed in self-assembled quantum rings ${ }^{13}$ (without magnetic field) clearly revealed a distinction from those spectra in quantum dots. This distinction is explained within the numerical simulations of the neutral exciton energy structure of quantum rings and lens-shaped quantum dots. ${ }^{14}$ Recently, there has been a renewed interest in the investigations of excitons in quantum rings ${ }^{10,15-17}$ in the presence of a magnetic field. It is argued that the Aharonov-Bohm effect is naturally associated with a net charge of a system as the coupling constant to the vector potential. A finite size of an exciton, as a composite particle, would possibly allow for a non-vanishing Aharonov-Bohm effect. The origin of the effect is the finite probability for electron and hole, created by a photon at the same point, to tunnel in the opposite directions and meet each other on the opposite side of the $1 \mathrm{D}$ ring. ${ }^{15}$

In Ref., ${ }^{16}$ for $2 \mathrm{D}$ quantum ring structures both with parabolic and the hard-wall confinement potentials, exciton binding energy and oscillator strength of the transitions were obtained by numerical diagonalization of the effective-mass Hamiltonian. Calculations performed using the parabolic basis with ring radius of $24 \mathrm{~nm}$ and width $21 \mathrm{~nm}$ did not reveal the Aharonov-Bohm oscillations. This negative result may be due to either of two reasons. First, perimeter of the ring is larger than the inverse decay length of the wave function of the internal motion of the electron and the hole, leading to the exponentially small magnitude of the Aharonov-Bohm effect. ${ }^{15}$ Second, there occurs a destructive interference of relatively many transverse eigenstates mixed by the Coulomb interaction. This analysis suggests that smaller and narrower rings are needed in experiments, which may lead to a picture of $1 \mathrm{D}$-like excitons. However, the authors of Ref. ${ }^{17}$ came to the conclusion that in the presence of Coulomb correlations, the Aharonov-Bohm effect of excitons as illustrated by the energy spectra and the oscillator strengths exists in a finite-width quantum rings with radius $20 \mathrm{~nm}$ and width $8 \mathrm{~nm}$. In wider rings (with increasing width in the range from 14 to $30 \mathrm{~nm}$ ) the non-simply-connected geometry is destroyed, causing a gradual suppression of the Aharonov-Bohm effect.

As suggested in Ref., ${ }^{10}$ the magnetic-field induced phase acquired by a polarized exciton rotating in a quantum ring leads to a switching between bright and dark exciton ground states, resulting in the suppression of the optical emission in certain windows of magnetic field.

In Ref., ${ }^{18}$ it was shown that the ground state energy of a negatively charged exciton in a quantum ring, unlike the neutral exciton, exhibits Aharonov-Bohm oscillations for any disk radius. The observed photoluminescence spectra of a single lithographically prepared quantum ring with radius $\sim 33 \mathrm{~nm}$, for magnetic field in the range from 0 to $5 \mathrm{~T}$, revealed the following features. ${ }^{19}$ The Zeeman-split lines corresponding to the exciton reveal a smooth shift to higher energies with increasing magnetic field (no Aharonov-Bohm oscillations are detected within the experimental accuracy), while those corresponding to the negatively charged trion reveal the Aharonov-Bohm oscillations. The measured photon energy from charged exciton recombination as a function of magnetic field is interpreted in terms of a transition from a negatively charged exciton to an electron in a model ring of infinitesimal width. The energy spectrum of the lowest-lying electron-hole states was studied in Ref. ${ }^{14}$ taking into account 
the quantum ring thickness and a coupling between heavy and light holes. That analysis implied that an optical detection of the Aharonov-Bohm effect in quantum rings could be realizable.

Recently, Aharonov-Bohm oscillations were detected in both the emission energy and intensity at elevated temperatures up to $180 \mathrm{~K}$ in type-II $\mathrm{ZnTe} / \mathrm{ZnSe}$ quantum dots. ${ }^{12}$

The analysis of the shape, size, and composition of SAQRs at the atomic scale performed by cross-sectional scanning-tunneling microscopy (X-STM) (see Refs. ${ }^{20,21}$ and a recent review ${ }^{22}$ ) demonstrated that the selfassembled quantum rings (SAQR) have an indium-rich craterlike shape, which is responsible for the ringlike properties of the SAQRs. Recently, the magnetic moment of a sample consisting of 29 layers of SAQRs has been measured at low temperature. By using an ultrasensitive torsion magnetometer in magnetic fields up to $15 \mathrm{~T}$, the oscillatory persistent current in SAQRs has been observed. ${ }^{3}$ It was explained using the theory of the electron energy spectra and the magnetization of a single-electron SAQR. ${ }^{2}$ The relevance of the two-electron systems for the interpretation of the Aharonov-Bohm oscillations in the persistent current in SAQRs has been discussed in Ref. ${ }^{4}$ using a numerical diagonalization of the two-electron Hamiltonian within a finite basis of anti-symmetrized wave functions for two electrons.

We extend the method, presented in Ref. ${ }^{4}$ for two-electron SAQRs, to excitons in SAQRs using the structural information from the X-STM measurements. ${ }^{20,21}$ We calculate the exciton energy spectra and the optical transition probabilities in SAQRs with a realistic anisotropic singly connected shape using a numerical diagonalization of the exciton Hamiltonian within a finite basis of wave functions for the electron and the hole. ${ }^{5}$

\section{EXCITON IN A STRAINED SAQR: THE MODEL}

In this Section, we present the main conceptual ingredients of our approach to analyze the energy spectrum and the probabilities of optical transitions for a neutral exciton in a strained $\operatorname{In}_{x} \mathrm{Ga}_{1-x} \mathrm{As} / \mathrm{GaAs}$ quantum ring with realistic parameters.

The Hamiltonian of an exciton in a strained quantum ring is

$$
H_{e x}=H_{e}+H_{h}+V_{\text {Coul }}\left(\mathbf{r}_{e}, \mathbf{r}_{h}\right),
$$

where $H_{e}\left(H_{h}\right)$ is the single-particle Hamiltonian of an electron (a hole), $V_{\text {Coul }}\left(\mathbf{r}_{e}, \mathbf{r}_{h}\right)$ describes the Coulomb interaction between an electron and a hole with radius-vectors $\mathbf{r}_{e}$ and $\mathbf{r}_{h}$, respectively. We consider here only heavy holes and treat them within the one-band model. The single-particle Hamiltonians in a strained ring have the form ${ }^{23,24}$

$$
\begin{gathered}
H_{e}=-\frac{\hbar^{2}}{2}\left(\nabla-\frac{e}{\hbar} \mathbf{A}\right) \frac{1}{m_{e}\left(\mathbf{r}_{e}\right)}\left(\nabla-\frac{e}{\hbar} \mathbf{A}\right)+U_{e}\left(\mathbf{r}_{e}\right)+\delta E_{e}\left(\mathbf{r}_{e}\right)-e V_{P}\left(\mathbf{r}_{e}\right), \\
H_{h}=-\frac{\hbar^{2}}{2}\left(\nabla+\frac{e}{\hbar} \mathbf{A}\right) \frac{1}{m_{h}\left(\mathbf{r}_{h}\right)}\left(\nabla+\frac{e}{\hbar} \mathbf{A}\right)-U_{h}\left(\mathbf{r}_{h}\right)-\delta E_{h}\left(\mathbf{r}_{h}\right)+e V_{P}\left(\mathbf{r}_{h}\right),
\end{gathered}
$$

where $m_{e}\left(\mathbf{r}_{e}\right)\left[m_{h}\left(\mathbf{r}_{h}\right)\right]$ is the conduction-electron [heavy-hole] mass and $\mathbf{A}=\mathbf{e}_{\varphi} B \rho / 2$ is the vector potential of the uniform magnetic field $\mathbf{B}=\mathbf{e}_{z} B . V_{e}\left(\mathbf{r}_{e}\right)\left[U_{h}\left(\mathbf{r}_{h}\right)\right]$ is the bottom of the conduction band [top of the valence band], determined by the In content $x$, in the absence of strain. The strain-induced shift of the conduction band

$$
\delta E_{\beta}=a_{\beta}\left(\varepsilon_{x x}+\varepsilon_{y y}+\varepsilon_{z z}\right)
$$

depends on the hydrostatic component of the strain tensor $\varepsilon_{j k}$. Here, $\beta=e, h$.

The shear strains give rise to the piezoelectric potential

$$
V_{P}(\mathbf{r})=-\frac{1}{4 \pi \varepsilon_{0} \varepsilon_{r}} \int \frac{\operatorname{div} \mathbf{P}}{\left|\mathbf{r}-\mathbf{r}^{\prime}\right|} d^{3} \mathbf{r}^{\prime}
$$

determined by the piezoelectric polarization $P_{i}=e_{i j k} \varepsilon_{j k}$, where for InAs and GaAs only the piezoelectric moduli $e_{123}=e_{213}=e_{312}$ are other than zero; $\varepsilon_{r}$ is the relative dielectric constant. The relevant material parameters are: $e_{123}^{\mathrm{InAs}}=0.045 \mathrm{C} / \mathrm{m}^{2}, e_{123}^{\mathrm{GaAs}}=0.16 \mathrm{C} / \mathrm{m}^{2}, a_{e}^{\mathrm{InAs}}=-5.08 \mathrm{eV}, a_{e}^{\mathrm{GaAs}}=-7.17 \mathrm{eV}, a_{h}^{\mathrm{InAs}}=1.00 \mathrm{eV}$, $a_{h}^{\text {GaAs }}=1.16 \mathrm{eV} \cdot{ }^{25}$ The band gap and the effective masses as well as the parameters $e_{123}$ and $a_{\beta}$ for $\operatorname{In}_{x} \mathrm{Ga}_{1-x} \mathrm{As}$ 
are taken from a linear interpolation between the corresponding values for InAs and GaAs. We further assume that for the conduction and valence bands in $\operatorname{In}_{x} \mathrm{Ga}_{1-x}$ As the band edge variations with $x$ in the absence of strain are to each other as $7: 3$.

The components of the strain tensor $\varepsilon_{j k}$ as well as the distribution of indium $x$ for a SAQR were calculated following a three-dimensional finite-element method of the elasticity theory. Using the tables of $\varepsilon_{j k}$ and $x$, used also in the calculations of the electron magnetic moment, we first numerically calculate and tabulate the distributions of the strain-induced shifts of the band edges (4) and of the piezoelectric potential (5).

Then the single-particle Schrödinger equations

$$
H_{\beta} \Psi^{(\beta)}(\mathbf{r})=E \Psi^{(\beta)}(\mathbf{r})
$$

are solved within the adiabatic approximation, using the Ansatz:

$$
\Psi^{(\beta)}(\mathbf{r})=\psi_{k}^{(\beta)}(z ; \rho, \varphi) \Phi_{k j}^{(\beta)}(\rho, \varphi),
$$

where the index $k$ numbers subbands due to the size quantization along the $z$-axis:

$$
\begin{aligned}
& {\left[-\frac{\hbar^{2}}{2} \frac{\partial}{\partial z} \frac{1}{m_{\beta}(\rho, \varphi, z)} \frac{\partial}{\partial z} \pm U_{\beta}(\rho, \varphi, z) \pm \delta E_{\beta}(\rho, \varphi, z) \mp e V_{P}(\rho, \varphi, z)\right] \psi_{k}^{(\beta)}(z ; \rho, \varphi) } \\
= & \mathcal{E}_{k}^{(\beta)}(\rho, \varphi) \psi_{k}^{(\beta)}(z ; \rho, \varphi) .
\end{aligned}
$$

In Eq. (3), the upper (lower) sign stands for conduction electrons (heavy holes), i.e. for $\beta=e(\beta=h)$. The Schrödinger equation (3) for the "fast" degree of freedom (corresponding to the motion along the $z$-axis) is solved numerically for each node of a two-dimensional grid in the $(\rho, \varphi)$-plane. As a result, we obtain the adiabatic potentials $\mathcal{E}_{k}^{(\beta)}(\rho, \varphi)$, tabulated on the aforementioned grid.

The Schrödinger equations for the "slow" degrees of freedom

$$
\left[-\frac{\hbar^{2}}{2}\left(\nabla_{\rho, \varphi} \mp \frac{e}{\hbar} \mathbf{A}\right) \frac{1}{m_{k}^{(\beta)}(\rho, \varphi)}\left(\nabla_{\rho, \varphi} \mp \frac{e}{\hbar} \mathbf{A}\right)+\mathcal{E}_{k}^{(\beta)}(\rho, \varphi)\right] \Phi_{k j}^{(\beta)}(\rho, \varphi)=E_{k j}^{(\beta)} \Phi_{k j}^{(\beta)}(\rho, \varphi)
$$

with the upper (lower) sign for $\beta=e(\beta=h)$, the adiabatic potentials $\mathcal{E}_{k}^{(\beta)}(\rho, \varphi)$, and the effective masses

$$
m_{k}^{(\beta)}(\rho, \varphi)=\int d z\left|\psi_{k}^{(\beta)}(z ; \rho, \varphi)\right|^{2} m_{\beta}(\rho, \varphi, z)
$$

determine the eigenstates of the in-plane motion, which are labeled by the index $j$. The Schrödinger equation (3) with an (anisotropic) adiabatic potential $\mathcal{E}_{k}^{(\beta)}(\rho, \varphi)$ and position-dependent effective mass $m_{k}^{(\beta)}(\rho, \varphi)$ cannot be solved analytically.

We find the eigenstates of the in-plane motion by a numerical diagonalization of the Hamiltonian, which enters the lhs of (3), in the basis of eigenfunctions of an auxiliary Hamiltonian with a constant effective mass $\bar{m}_{k}^{(\beta)}$ and a simplified adiabatic potential. We chose this simplified adiabatic potential in the form

$$
\overline{\mathcal{E}}_{k}^{(\beta)}(\rho)=v_{k}^{(\beta)}+w_{k}^{(\beta)} \rho^{2} .
$$

Since the potential (4) is isotropic, the corresponding in-plane eigenfunctions can be written down as

$$
\bar{\Phi}_{k n L}^{(\beta)}(\rho, \varphi)=\bar{\chi}_{k n L}^{(\beta)}(\rho) \mathrm{e}^{i L \varphi},
$$

where $n$ labels the radial solutions.

Inserting into (3) the adiabatic potential (4) instead of $\mathcal{E}_{k}^{(\beta)}(\rho)$, a constant effective mass $\bar{m}_{k}^{(\beta)}$, and wave functions in the form of (5), one obtains the one-dimensional Schrödinger equation

$$
\left(\bar{\chi}_{k n L}^{(\beta)}\right)^{\prime \prime}+\frac{1}{\rho}\left(\bar{\chi}_{k n L}^{(\beta)}\right)^{\prime}+\left[\frac{2 \bar{m}_{k}^{(\beta)}}{\hbar^{2}}\left(E_{k n L}^{(\beta)}-v_{k}^{(\beta)}\right)+\frac{L}{l^{2}}-\frac{L^{2}}{\rho^{2}}-\left(\alpha_{k}^{(\beta)} \rho\right)^{2}\right] \bar{\chi}_{k n L}^{(\beta)}=0,
$$


where $l=\sqrt{\hbar /(e B)}$ is the magnetic length and

$$
\alpha_{k}^{(\beta)}=\sqrt{\frac{1}{4 l^{4}}+\frac{2 \bar{m}_{k}^{(\beta)} w_{k}^{(\beta)}}{\hbar^{2}}} .
$$

The eigenfunctions of Eq. (6) are

$$
\bar{\chi}_{k n L}^{(\beta)}(\rho)=C_{k n L} \rho^{|L|} \exp \left(-\frac{\alpha_{k}^{(\beta)} \rho^{2}}{2}\right) L_{n}^{(|L|)}\left(\alpha_{k}^{(\beta)} \rho^{2}\right), \quad n=0,1,2, \ldots
$$

where $L_{n}^{(m)}(x)$ are generalized Laguerre polynomials and $C_{k n L}$ are normalization constants. The corresponding eigenenergies are

$$
E_{k n L}^{(\beta)}=v_{k}^{(\beta)}+\frac{\hbar^{2}}{\bar{m}_{k}^{(\beta)}}\left[\left(n+\frac{1}{2}-\frac{L}{2}\right) \frac{1}{l^{2}}+|L| \alpha_{k}^{(\beta)}\right] .
$$

We are interested in the lowest states of an electron and a hole in the quantum ring. Therefore, we restrict our calculations to the states in the lowest subband of the (strong) size-quantization along the $z$-axis (i.e., we consider states with $k=1$ ). For each value of the applied magnetic field, the electron and hole eigenstates in the quantum ring are found by numerical diagonalization of the adiabatic Hamiltonian, which enters the lhs of (3), in a finite basis of the in-plane wave functions $\bar{\Phi}_{1 n L}^{(\beta)}(\rho, \varphi)$, given by Eq. (5) with $\bar{\chi}_{1 n L}^{(\beta)}(\rho)$ of Eq. (7). The aforementioned finite basis includes functions with $n=0, \ldots, n_{\max }$ and $L=-L_{\max }, \ldots, L_{\max }$. In the present calculations, we use $n_{\max }=15$ and $L_{\max }=12$, the parameters of the auxiliary Hamilatonian are taken as $v_{1}^{(e)}=650 \mathrm{meV}, w_{1}^{(e)}=0.43 \mathrm{meV} / \mathrm{nm}^{2}, \bar{m}_{1}^{(e)}=0.053 m_{0}, v_{1}^{(h)}=210 \mathrm{meV}, w_{1}^{(h)}=0.31 \mathrm{meV} / \mathrm{nm}^{2}, \bar{m}_{1}^{(h)}=0.5 m_{0}$.

As a result of diagonalization, we obtain the energies $E_{1 j}^{(\beta)}$ and the wavefunctions

$$
\begin{aligned}
\Psi_{1 j}^{(\beta)}(\mathbf{r}) & =\psi_{1}^{(\beta)}(z ; \rho, \varphi) \sum_{L=-L_{\max }}^{L_{\max }} \sum_{n=0}^{n_{\max }} a_{1 j n L} \bar{\chi}_{1 n L}^{(\beta)}(\rho) \mathrm{e}^{i L \varphi} \\
& =\psi_{1}^{(\beta)}(z ; \rho, \varphi) \sum_{L=-L_{\max }}^{L_{\max }} \chi_{1 j L}^{(\beta)}(\rho) \mathrm{e}^{i L \varphi}
\end{aligned}
$$

of the lowest electron and hole states in the quantum ring as a function of the applied magnetic field. Here,

$$
\chi_{1 j L}^{(\beta)}(\rho)=\sum_{n=0}^{n_{\max }} a_{1 j n L} \bar{\chi}_{1 n L}^{(\beta)}(\rho) .
$$

The index $j=1,2,3, \ldots$ labels states in the order of increasing energy.

In order to find exciton eigenstates, we start with constructing the basis functions, which describe a noninteracting eh-pair:

$$
\Psi_{j_{e} j_{h}}^{(e h)}\left(\mathbf{r}_{e}, \mathbf{r}_{h}\right)=\Psi_{1 j_{e}}^{(e)}\left(\mathbf{r}_{e}\right) \Psi_{1 j_{h}}^{(h)}\left(\mathbf{r}_{h}\right)
$$

In the present calculations, this basis contains 144 functions, which correspond to $j_{e}, j_{h}=1, \ldots, j_{\max }$ with $j_{\max }=12$. Then we diagonalize the Hamiltonian (1) in the above basis, looking for the exciton wavefunctions in the form

$$
\Psi_{J}^{(e x)}\left(\mathbf{r}_{e}, \mathbf{r}_{h}\right)=\sum_{j_{e}, j_{h}=1}^{j_{\max }} A_{J j_{e} j_{h}} \Psi_{j_{e} j_{h}}^{(e h)}\left(\mathbf{r}_{e}, \mathbf{r}_{h}\right)
$$


The most time-consuming stage is the calculation of matrix elements of the Coulomb interaction $V_{\text {Coul }}\left(\mathbf{r}_{e}, \mathbf{r}_{h}\right)$. Analogously to Ref., ${ }^{2}$ it is convenient to represent these matrix elements as

$$
\begin{aligned}
\left(V_{\mathrm{Coul}}\right)_{j_{e 2}, j_{h 2}, j_{e 1}, j_{h 1}}= & \int d^{3} \mathbf{r}_{e} \int d^{3} \mathbf{r}_{h}\left[\Psi_{j_{e 1} j_{h 1}}^{(e h)}\left(\mathbf{r}_{e}, \mathbf{r}_{h}\right)\right]^{*} V_{\mathrm{Coul}}\left(\mathbf{r}_{e}, \mathbf{r}_{h}\right) \Psi_{j_{e 1} j_{h 1}}^{(e h)}\left(\mathbf{r}_{e}, \mathbf{r}_{h}\right) \\
= & \sum_{\substack{L_{e 1}, L_{h 1}, L_{e 2}, L_{h 2}=-L_{m a x} \\
L_{m a x}}}^{\infty} d \rho_{e} \int_{0}^{\infty} d \rho_{h}\left[\chi_{1 j_{e 2} L_{e 2}}^{(e)}\left(\rho_{e}\right) \chi_{1 j_{h 2} L_{h 2}}^{(h)}\left(\rho_{h}\right)\right]^{*} \\
& \times \chi_{1 j_{e 1} L_{e 1}}^{(e)}\left(\rho_{e}\right) \chi_{1 j_{h 1} L_{h 1}}^{(h)}\left(\rho_{h}\right) S_{L_{e 1}-L_{e 2}, L_{h 1}-L_{h 2}}\left(\rho_{e}, \rho_{h}\right),
\end{aligned}
$$

where

$$
\begin{aligned}
S_{\Delta L_{e}, \Delta L_{h}}\left(\rho_{e}, \rho_{h}\right)= & -\frac{e^{2} \rho_{e} \rho_{h}}{4 \pi \varepsilon_{0} \varepsilon_{r}} \int_{0}^{2 \pi} d \varphi_{e} \int_{0}^{2 \pi} d \varphi_{h} \int_{-\infty}^{\infty} d z_{e} \int_{-\infty}^{\infty} d z_{h}\left|\psi_{1}^{(e)}\left(z_{e} ; \rho_{e}, \varphi_{e}\right)\right|^{2} \\
& \times\left|\psi_{1}^{(h)}\left(z_{h} ; \rho_{h}, \varphi_{h}\right)\right|^{2} \frac{\mathrm{e}^{i \Delta L_{e} \varphi_{e}+i \Delta L_{h} \varphi_{h}}}{\sqrt{\rho_{e}^{2}+\rho_{h}^{2}-2 \rho_{e} \rho_{h} \cos \left(\varphi_{e}-\varphi_{h}\right)+\left(z_{e}-z_{h}\right)^{2}}}
\end{aligned}
$$

Importantly, the integrals $S_{\Delta L_{e}, \Delta L_{h}}\left(\rho_{e}, \rho_{h}\right)$ do not depend on the magnetic field. Therefore, we first tabulate $S_{\Delta L_{e}, \Delta L_{h}}\left(\rho_{e}, \rho_{h}\right)$. Then for each magnetic field $B$ the matrix elements (11) are calculated and the lowest exciton states are found by numerical diagonalization of the Hamiltonian (1) in the basis (9).

\section{DISCUSSION OF RESULTS}

\subsection{The effect of the Coulomb interaction in the isotropic SAQRs}
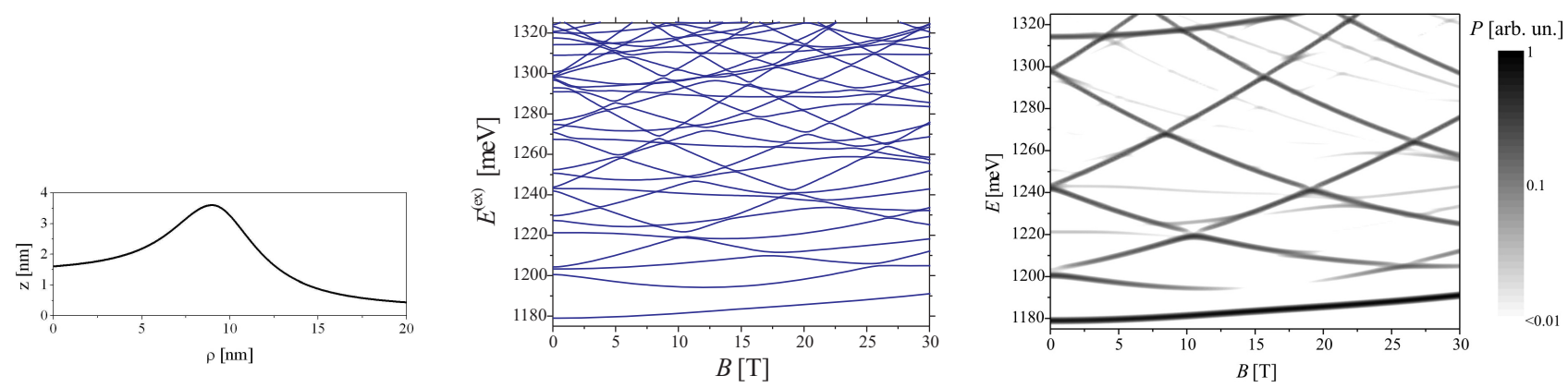

Figure 1. Middle panel: Energies of the lowest dipole active exciton states in an axially symmetric $\operatorname{In}_{0.55} \mathrm{Ga}_{0.45} \mathrm{As} / \mathrm{GaAs}$ ring-like structure as a function of the applied magnetic field. Lhs panel: Shape of the structure. Rhs panel: Spectral distribution of the optical transition probabilities $P$ for an exciton as a function of the applied magnetic field $(\Gamma=1 \mathrm{meV})$.

The shape of the axially isotropic ring-like structure with $x=0.55$, assumed in our calculations (without strain), is shown in the lhs panel in Fig. 1. We deal only with the states, which belong to the lowest subband of the (strong) size quantization of the particle motion along the growth axis. Due to strong selection rules, applicable for the highly symmetric structure under consideration, only a small fraction of the states of the non-interacting eh-pair are dipole active. Namely, these are the states with zero envelope angular momentum of the eh-pair, $L_{e}+L_{h}=0$. Owing to different quantization of the radial motion for electrons and holes, dipole transitions to states with $n_{e} \neq n_{h}$, strictly speaking, are not forbidden, although their probabilities are significantly lower than those for transitions to states with $n_{e}=n_{h}$. Dipole active energy levels of the exciton are plotted in the middle panel in Fig. 1. Due to the electron-hole Coulomb interaction, the lowest exciton states shift downwards by $16 \mathrm{meV}$ as compared to the non-interacting eh-pair. As seen from the middle panel in Fig. 1, the electron-hole Coulomb interaction leads to anticrossing for the lowest two dipole-active energy levels (for a non-interacting eh-pair, the corresponding levels cross each other). The reason is that, in the presence of the 
electron-hole Coulomb interaction, only the envelope angular momentum of the exciton, $L_{e}+L_{h}$ (but not $L_{e}$ and $L_{h}$ separately) remains a good quantum number. In other words, the Coulomb interaction mixes eh-states with the same $L_{e}+L_{h}$ and different $L_{e}\left(=-L_{h}\right), n_{e}, n_{h}$. Of course, such a mixing is especially strong for energy levels, which are close to each other. This mixing of states due to the electron-hole Coulomb interaction is accompanied by a redistribution of oscillator strengths (usually, in favor of lower-lying states). This is illustrated on the rhs panel in Fig. 1, where we plot the spectral distributions of the calculated probabilities $P$ for optical transitions to different states of an exciton. In order to enhance visualization, a small Gaussian broadening $(\sim 1$ meV) is introduced for all the energy levels. In the energy range, which corresponds to anticrossing of the lowest two dipole active energy levels, the upper of these two levels becomes "dark", while the transition probability $P$ for the lower level increases.

\subsection{The effects of strain and of the In content in axially isotropic SAQRs}
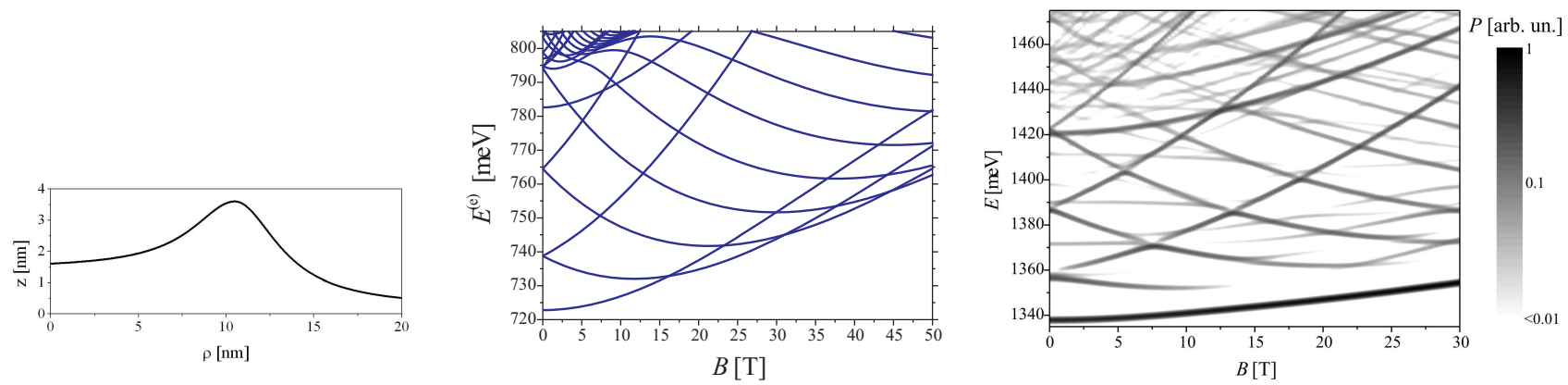

Figure 2. Middle panel: Energies of the lowest electron states in a strained axially symmetric $\operatorname{In}_{0.65} \mathrm{Ga}_{0.35} \mathrm{As} / \mathrm{GaAs}$ ring-like structure as a function of the applied magnetic field. Lhs panel: Shape of the structure. Rhs panel: Spectral distribution of the optical transition probabilities $P$ for an exciton as a function of the applied magnetic field $(\Gamma=1 \mathrm{meV})$.

We analyzed the electron energy spectrum, the magnetization, and the optical-transition probabilities for excitons in strained axially symmetric ring-like structures (see the lhs panel in Fig. 2) with different In concentration in the In-rich region $(x=0.55, x=0.65$ and $x=0.7)$. We neglect the strain-induced piezoelectric potential, because the piezoelectric field actually has negligible effect on the electron states in SAQRs of typical sizes considered here (see Fig. $3 \mathrm{~b}$ in Ref. ${ }^{2}$ for quantitative details).

The strain-induced flattening of the adiabatic potential makes the structure under consideration "disk-like" rather than "ring-like". However, due to an increase of the In content $x$, the depth of the adiabatic potential well in the In-rich region significantly increases both for an electron and for a hole. This results, in particular, in a decrease of the electron ground-state energy shown on the middle panel in Fig. 2, where energies are counted from the bottom of the conduction band in unstrained bulk InAs. Consequently, an increase of the In content $x$ leads to an increase of the energy spacing between the lowest electron energy level and the bottom of the continuum of states in the wetting layer: at zero applied magnetic field, this spacing is about $55 \mathrm{meV}$ for $x=0.55$ and about $75 \mathrm{meV}$ for $x=0.65$. In the rhs panel of Fig. 2, we show spectral distributions of the calculated probabilities $P$ for optical transitions to different states of an exciton in a strained $\operatorname{In}_{0.65} \mathrm{Ga}_{0.35} \mathrm{As} / \mathrm{GaAs}$ ring-like structure. At relatively low magnetic fields $B$, a complicated pattern of $P$ starts for energies $\geq 100$ meV above the lowest exciton state. This energy region actually corresponds to continuum states. The energy of the lowest optically active exciton state at $B=0$ constitutes $1338 \mathrm{meV}$ (cp. with the respective value of $1179 \mathrm{meV}$ for an unstrained ring-like structure in the middle panel on Fig. 1). An increase of the In content to $x=0.7$ leads to a good agreement of the model with the experimental data ${ }^{26}$ in what concerns the position of the lowest exciton energy level and the energy difference between this level and the bottom of the continuum of states in the wetting layer.

\subsection{The effect of the anisotropy of a SAQR}

We calculated the probabilities $P$ of optical transitions involving different states of an exciton in the anisotropic ring-like structure with $x=0.7$. The anisotropy of this structure is characterized by the set of parameters 

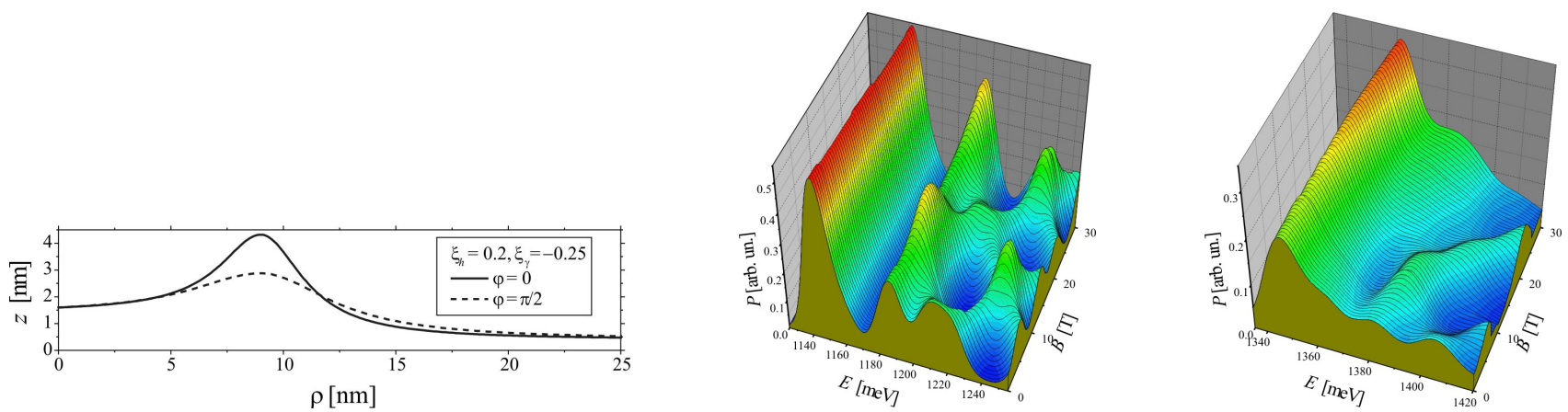

Figure 3. Middle panel: Broadened spectral distributions of $P$ for an exciton in the anisotropic unstrained $\mathrm{In}_{0.7} \mathrm{Ga}_{0.3} \mathrm{As} / \mathrm{GaAs}$ ring-like structure with the anisotropy parameters $\xi_{h}=0.2, \xi_{\gamma}=-0.25$ and with $\Gamma=10 \mathrm{meV}$. Lhs panel: Shape of the structure. Rhs panel: Broadened spectral distributions of $P$ for an exciton in the anisotropic strained $\mathrm{In}_{0.7} \mathrm{Ga}_{0.3} \mathrm{As} / \mathrm{GaAs}$ ring-like structure $\left(\xi_{h}=0.2, \xi_{\gamma}=0\right.$ and $\left.\Gamma=10 \mathrm{meV}\right)$.

(cf. $\left.^{2}\right) \quad \xi_{h}=0.2$ and $\xi_{\gamma}=-0.25$. Other relevant parameters are $R=9 \mathrm{~nm}, h_{0}=1.6 \mathrm{~nm}, h_{M}=3.6 \mathrm{~nm}$, $h_{\infty}=0.4 \mathrm{~nm}, \gamma_{0}=\gamma_{\infty}=3 \mathrm{~nm}, \xi_{R}=0$. On the lhs panel in Fig. 3 we show the cross-sections of the structure under consideration in the $x z$ - and $y z$-planes.

In the present calculations, we take into account 8 lowest electron states and 20 lowest hole states. The number of hole states in the relevant energy interval $(\geq 120 \mathrm{meV}$ above the lowest hole state) is significantly larger than 20 (in fact, it is $\geq 60$ ). However, the results of our analysis (not shown here) imply that the probabilities of optical transitions between the lowest electron states and highly excited hole states are negligibly small because the corresponding hole wave functions are almost exactly orthogonal to the wave functions of the lowest electron states. Therefore, it is safe to assume that the use of the aforementioned limited basis of single-particle states is sufficient for an adequate description of the function $P(E, B)$ for an exciton. That basis allows us to perform diagonalization of the exciton Hamiltonian within a reasonably short computation time.

In the case of relatively small energy-level broadening $(\Gamma=1 \mathrm{meV})$, the Coulomb interaction leads to a mixing of the two lowest quasi-degenerate "bright" eh-states (of course, also other "bright" and "dark" eh-states contribute to the resulting exciton wave functions) and to a strong anticrossing effect for the resulting two energy levels. Moreover, due to the Coulomb interaction the oscillator strengths of these two exciton levels are significantly redistributed in favor of the lowest level. Therefore, despite a relatively large splitting between these two exciton energy levels, the upper one is practically unresolvable in the case of an appreciable energy-level broadening, $\Gamma=10 \mathrm{meV}$ (see the middle panel in Fig. 3). This fact indicates that - as compared to the model of axially symmetric rings, studied in the previous subsections, - the model of anisotropic ring-like structures is more consistent with the experimental data. ${ }^{26}$

\subsection{The effect of the anisotropy of a strained SAQR}

Finally, we consider an anisotropic $\operatorname{In}_{0.7} \mathrm{Ga}_{0.3} \mathrm{As} / \mathrm{GaAs}$ model structure in accordance with Ref. ${ }^{2}$ using the following anisotropy parameters $\xi_{R}=0, \xi_{h}=0.2$, and $\xi_{\gamma}=0$. Other relevant parameters are: $R=9 \mathrm{~nm}$, $h_{0}=1.6 \mathrm{~nm}, h_{M}=3.6 \mathrm{~nm}, h_{\infty}=0.4 \mathrm{~nm}, \gamma_{0}=\gamma_{\infty}=3 \mathrm{~nm}$.

Due to strain the zero-field splitting between the second and third energy levels (which would correspond to the states with $L= \pm 1$ in an isotropic ring) significantly decreases, while the transition fields, which correspond to interchange of the lowest two energy levels of an electron, significantly increase. The first effect is caused by the fact that strain smoothes angular variations of the adiabatic potential for an electron, so that the influence of the shape anisotropy on the electron angular motion becomes weaker. The second effect is due to the strain-induced lowering of the potential barrier at the center of the ring, which results in a decrease of the electron effective radius in the ring-like structure under consideration. For the structure under analysis here, the presence of strain leads not only to an increase of transition fields for the Aharonov-Bohm oscillations but also to an appreciable increase of the oscillation amplitude. The latter is caused by the aforementioned strain-induced smoothing of 

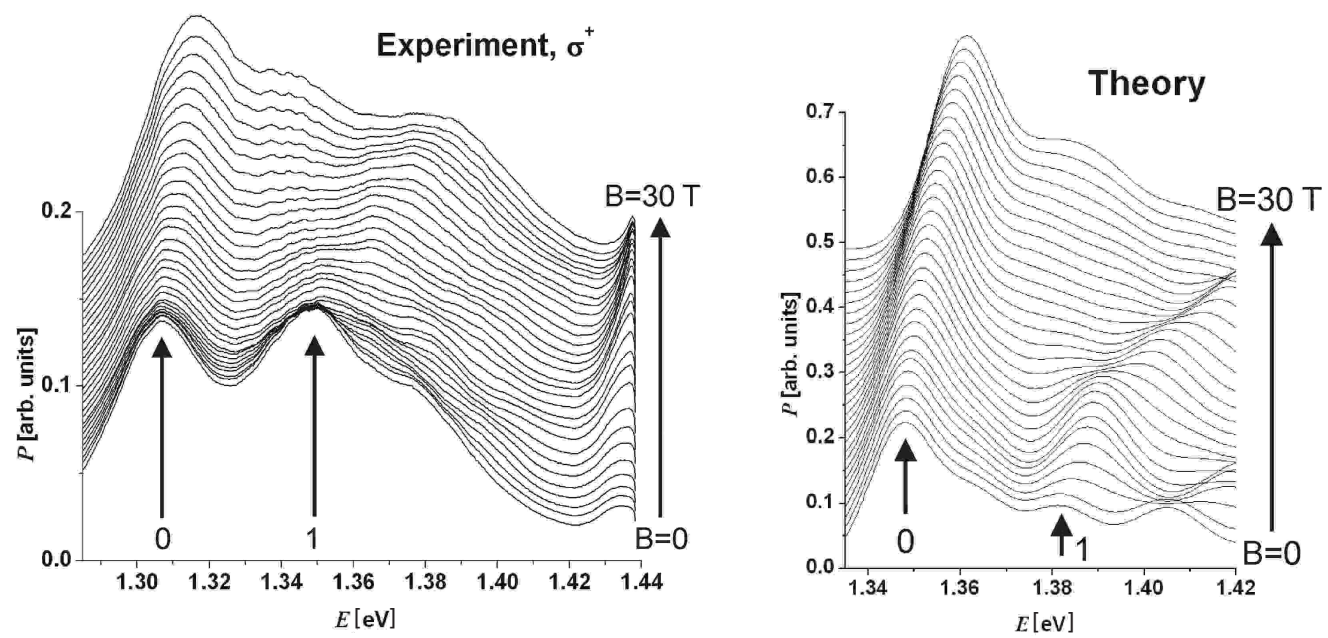

Figure 4. Lhs panel: Experimental $\sigma^{+}$photoluminescence spectra of self-assembled InGaAs/GaAs quantum rings in magnetic fields from $0 \mathrm{~T}$ (the lowest curve) to $30 \mathrm{~T}$ (the highest curve) with the step $\Delta B=1 \mathrm{~T}$. The two lowest peaks at $B=0$ are indicated with labels " 0 " (at $1.30735 \mathrm{eV}$ ) and "1" (at $1.34945 \mathrm{eV}$ ), corresponding to the peaks " $0 "$ and "1" in the rhs panel. Rhs panel: theoretical spectra of the optical transition probabilities (cf. the rhs panel in Fig. 3).

the potential barriers for the angular motion of an electron, which are rather high in the unstrained ring (due to a relatively large value of $\xi_{h}$ and the absence of "compensating" variations of the rim width, i.e. $\xi_{\gamma}=0$ ).

The spectral distribution of the calculated probabilities $P(E)$ as a function of the applied magnetic field $B$ is shown in the rhs panel in Fig. 3. When compared to the anisotropic unstrained $\operatorname{In}_{0.7} \mathrm{Ga}_{0.3} \mathrm{As} / \mathrm{GaAs}$ structure and to the strained $\mathrm{In}_{0.55} \mathrm{Ga}_{0.45} \mathrm{As} / \mathrm{GaAs}$ structure, for the strained structure, analyzed here, positions of the most pronounced peaks of $P(E)$ for an exciton are more consistent with the experiment (see Fig. 4).

At the same time, a better agreement between the calculated and experimental results seems to imply a model ring-like structure with a higher and wider rim as well as with a thinner wetting layer, where the In content is reduced as compared to the In content in the rim.

\section{CONCLUSIONS}

We have calculated electron and exciton spectra and optical transition probabilities for various models of the $\mathrm{In}_{x} \mathrm{Ga}_{1-x} \mathrm{As} / \mathrm{GaAs}$ SAQRs. Quantitative parameters, which characterize the behavior of exciton energy spectra and optical-transition probabilities as a function of the applied magnetic field, are very sensitive to the details of the quantum-ring shape. Spectral distributions of the probabilities of optical transitions between the exciton vacuum and one-exciton states as a function of the applied magnetic field are characterized by rich patterns, which significantly depend on shape, size and composition of the ring-like nanostructures. Our analysis of the photoluminescence spectrum in magnetic fields up to $30 \mathrm{~T}$ shows that the excitonic properties strongly depend on the anisotropic shape, size, composition and strain of the SAQRs.

\section{ACKNOWLEDGMENTS}

We acknowledge collaboration with P. Offermans, I. M. A. Bominaar-Silkens and U. Zeitler as well as support by the FWO-V (Belgium), the NanoNed program of the Dutch Ministry of Economic Affairs (The Netherlands), the projects NANINPHO-QD MICINN (TEC2008-06756-C03-01), NANOCOMIC CAM (S-505/ESP/000200) and Consolider QUOIT MEC (CSD2006-00019) (Spain), the INTAS project no. 05-104-7656 and the EC Network of Excellence SANDiE, contract No. NMP4-CT-2004-500101. 


\section{REFERENCES}

[1] Y. Aharonov and D. Bohm, "Significance of electromagnetic potentials in the quantum theory", Phys. Rev. 115, pp. 485-491, 1959.

[2] V. M. Fomin, V. N. Gladilin, S. N. Klimin, J. T. Devreese, N. A. J. M. Kleemans, and P. M. Koenraad, "Theory of electron energy spectrum and Aharonov-Bohm effect in self-assembled $\operatorname{In}_{x} \mathrm{Ga}_{1-x}$ As quantum rings in GaAs", Phys. Rev. B 76, 235320, pp. 1-8, 2007.

[3] N. A. J. M. Kleemans, I. M. A. Bominaar-Silkens, V. M. Fomin, V. N. Gladilin, D. Granados, A. G. Taboada, J. M. García, P. Offermans, U. Zeitler, P. C. M. Christianen, J. C. Maan, J. T. Devreese, and P. M. Koenraad, "Oscillatory persistent currents in self-assembled quantum rings", Phys. Rev. Lett. 99, 146808, pp. 1-4, 2007.

[4] V. M. Fomin, V. N. Gladilin, J. T. Devreese, N. A. J. M. Kleemans, and P. M. Koenraad, "Energy spectra and oscillatory magnetization of two-electron self-assembled $\mathrm{In}_{x} \mathrm{Ga}_{1-x}$ As quantum rings in GaAs", Phys. Rev. B 77, 205326, pp. 1-5, 2008.

[5] V. M. Fomin, V. N. Gladilin, J. T. Devreese, N. A. J. M. Kleemans, M. Bozkurt, and P. M. Koenraad, "Electron and exciton energy spectra in self-assembled InGaAs/GaAs ring-like nanostructures", Physica Status Solidi (b), 245, pp. 2657-2661, 2008.

[6] A. V. Chaplik, "Magnetoexcitons in quantum rings and in antidots", JETP Lett. 62, pp. 900-904, 1995.

[7] A. V. Kalameitsev, A. O. Govorov, and V. M. Kovalev, "Magnetoexcitons in type-II quantum dots", JETP Lett. 68, pp. 669-672, 1998.

[8] K. L. Janssens, B. Partoens, and F. M. Peeters, "Magnetoexcitons in planar type-II quantum dots in a perpendicular magnetic field", Phys. Rev. B 64, 155324, pp. 1-9, 2001.

[9] A. V. Chaplik, "Aharonov-Bohm effect for composite particles and collective excitations", JETP Lett. 75, pp. 292-296, 2002.

[10] A. O. Govorov, S. E. Ulloa, K. Karrai, and R. J. Warburton, "Polarized excitons in nanorings and the optical Aharonov-Bohm effect", Phys. Rev. B 66, 081309(R), pp. 1-4, 2002.

[11] M. Grochol, F. Grosse, and R. Zimmermann, "Optical exciton Aharonov-Bohm effect, persistent current, and magnetization in semiconductor nanorings of type I and II", Phys. Rev. B 74, 115416, pp. 1-12, 2006.

[12] I. R. Sellers, V. R. Whiteside, I. L. Kuskovky, A. O. Govorov, and B. D. McCombe, "Aharonov-Bohm excitons at elevated temperatures in type-II ZnTe/ZnSe quantum dots", Phys. Rev. Lett. 100, 136405, pp. $1-4,2008$.

[13] H. Petterson, R. J. Warburton, A. Lorke, K. Karrai, J. P. Kotthaus, J. M. García, and P. M. Petroff, "Excitons in self-assembled quantum ring-like structures", Physica E 6, pp. 510-513, 2000.

[14] J. I. Climente, J. Planelles, and W. Jaskólski, "Magneto-optical transitions in nanoscopic rings", Phys. Rev. $B$ 68, 075307, pp. 1-8, 2003.

[15] R. A. Römer and M. E. Raikh, "Aharonov-Bohm effect for an exciton", Phys. Rev. B 62, pp. 7045-7049, 2000 .

[16] J. Song and S. E. Ulloa, "Magnetic field effects on quantum ring excitons", Phys. Rev. B 63, 125302, pp. $1-9,2001$.

[17] H. Hu, J.-L. Zhu, D.-J. Li, and J.-J. Xiong, "Aharonov-Bohm effect of excitons in nanorings", Phys. Rev. $B$ 63, 195307, pp. 1-11, 2001.

[18] M. Korkusiński, P. Hawrylak, and M. Bayer, "Negatively Charged Exciton on a Quantum Ring", Phys. Stat. Sol. (b) 234, pp. 273-282, 2002.

[19] M. Bayer, M. Korkusinski, P. Hawrylak, T. Gutbrod, M. Michel, and A. Forchel, "Optical detection of the Aharonov-Bohm effect on a charged particle in a nanoscale quantum ring", Phys. Rev. Lett. 90, 186801, pp. $1-4,2003$.

[20] P. Offermans, P. M. Koenraad, J. H. Wolter, D. Granados, J. M. García, V. M. Fomin, V. N. Gladilin, and J. T. Devreese, "Atomic-scale structure of self-assembled $\operatorname{In}(\mathrm{Ga})$ As quantum rings in GaAs", Appl. Phys. Lett. 87, 131902, pp. 1-3, 2005.

[21] P. Offermans, P. M. Koenraad, J. H. Wolter, D. Granados, J. M. García, V. M. Fomin, V. N. Gladilin, and J. T. Devreese, "Atomic-scale structure and formation of self-assembled $\operatorname{In}(\mathrm{Ga})$ As quantum rings", Physica E 32, pp. 41-45, 2006. 
[22] V. M. Fomin and L. F. Chibotaru, "Electronic properties of self-assembled nanostructures: Theoretical modeling on the base of the scanning tunneling microscopy characterization", Journal of Nanoelectronics and Optoelectronics 4, pp. 1-17, 2009.

[23] M. Grundmann, O. Stier, and D. Bimberg, "InAs/GaAs pyramidal quantum dots: Strain distribution, optical phonons, and electronic structure", Phys. Rev. B 52, pp. 11969-11981, 1995.

[24] J. A. Barker and E. P. O'Reilly, "Theoretical analysis of electron-hole alignment in InAs-GaAs quantum dots", Phys. Rev. B 61, pp. 13840-13851, 2000.

[25] C. G. Van de Walle, "Band lineups and deformation potentials in the model-solid theory", Phys. Rev. B 39, pp. 1871-1883, 1989.

[26] N. A. J. M. Kleemans, H. C. M. van Genuchten, M. Bozkurt, P. M. Koenraad, J. H. Blokland, P. C. M. Christianen, J. C. Maan, A. G. Taboada, D. Granados, J. M. García, V. M. Fomin, V. N. Gladilin, and J. T. Devreese, "Excitonic behavior in self-assembled InAs/GaAs quantum rings in high magnetic fields" (to be published). 